\title{
El Estudio de las Infecciones Congénitas por Toxoplasma Gondii y Trypanosoma Cruzi
}

\author{
Dra. Erica Thiermann O.; Dra. Patricia Muñoz C. del V.; T.M. Myriam Lorca H.; \\ Dr. Antonio Atías M. ${ }^{1}$. \\ The Surveillance of Congenital Infections \\ With T. Gondii and T. Cruzi
}

\begin{abstract}
The usefulness of systematic serological screening of congenital toxoplasmosis and Chagas' disease in our country is discussed on the basis of 855 cases, which had been studied in three places of Chile (Santiago, Vicuña and Ovalle). Prevalence of maternal toxoplasmosis reaches $35 \%$ in Santiago, $29 \%$ in Vicuña and $26 \%$ in Ovalle. High titers, indicating early infection of the mothes, wese found in one case, but congenital toxoplasmosis was not considered in her child because his serological tests became finally negative. Prevention of congenital toxoplasmosis by screening of women during pregnancy (at a great scale) would be far too expansive, technically difficult and of doubtful usefulness in our country. Instead, early recognition of the infection by serological examination of suspected newborns is recommended. Chagas disease was demonstrated in 4 of the 855 cases, three of them being of congenital origin. Prevalence of maternal infection was low in Santiago (2.7\%); but congenital Chagas' disease could be detected in 2 out of 10 infants born to serologically positive mothers. However, in the high endemic areas, where T. cruzi-infection reaches $11.8 \%$ (Vicuña) and $17.9 \%$ (Ovalle), only one congenital infection was demonstrated in a total of 61 newborns considered to be a risk. Additional information (by systematic screening of mothers and newborns in areas of low and high endemicy) is needed in order to clarify the regional differences observed in the present study. A surveillance program for congenital Chagas' disease is considered to be technically feasible in Chile. These studies would be restricted to endemic zones, the number of eases which are at risk for congenital infection would be manageable ( $2 \%$ to $20 \%$ of the total populations studied) and detection of these cases would be obtained by serological examination of one sample per case. The strategies for studying both congenital infections are given in detail.

Key words: American trypanosomiasis. Chagas' disease. Congenital. Maternal. Serological screening. Toxoplasma gondii. Toxoplasmosis. Transplacental transmission. Trypanosoma cruzi. Trypanosomiasis, American. Epidemiology. Prevention. Follow-up).
\end{abstract}

Las infecciones congénitas por Toxoplasma gondii y Trypanosoma cruzi constituyen un pro. blema importante en Pediatria. Dichas parasitosis presentan aspectos clínicos comunes que justificarian un enfoque conjunto:

En ambos casos se tropieza con dificultades en el reconocimiento oportuno de las infecciones maternas, ya que las embarazadas infectadas, generalmente no presentan síntomas evidentes.

Ambos parásitos pueden producir infecciones fetales agudas, a menudo graves. Sin embargo, dichos casos "clásicos", clinicamente manifiestos en el recién nacido, son poco frecuentes y sus síntomas, relativamente característicos inducen a estudiar su etiología $y$, por ende, a tratarlos.

El problema de mayor relevancia estaría cons-

1. Unidad de Parasitología, Dívisión Ciencias Médicas Occidente. Facultad de Medicina. Universidad de Chile.

Proyecto finsnciado parcialmente por Proyecto 81/91, D.1.U.C. tituido en ambos casos por aquellas infecciones intrauterinas que, al transcurrir en forma más sutil, no producen sintomas evidentes en el re. cièn nacido. Dichas infecciones congénitas, inaparentes en el nacimiento, pueden evolucionar si no se les trata oportunamente $y$, en estos casos, suelen aparecer síntomas tardíos o secuelas irreparables en períodos más avanzados de la vida ${ }^{1}$. $\mathrm{La}$ incidencia y el alcance clinico de dicho fenómeno no ha podido precisarse con certeza para la toxoplasmosis y parece aun más enigmático con respecto a la enfermedad de Chagas. En ambos casos se otorga primordial importancia a la pesquisa precoz de las infecciones parasitarias leves ("asintomáticas") en los recién nacidos, con el fin de tratarlos a la brevedad posible.

El reconocimiento oportuno de este tipo de infecciones congénitas sólo puede lograrse a través de estudios serológicos, practicados en forma sistemátjca, en embarazadas, recién nacidos $o$ ambos. Tales estudios pueden realizarse en forma simultánea para toxoplasmosis y enfermedad de Chagas, sin embargo, T. gondii y T. cruzi difieren 
en algunos aspectos, que deben tenerse presente al planificar los estudios mencionados.

El T. gondii es un parásito cosmopolita cuya importancia clínica ha sido bien estudiada aclarando aspectos relevantes de su transmisión transplacentaria y sus consecuencias para el recién nacido R.N.2- 3-4. La Infección congénita es la consecuencia de una primoinfección materna, adquirida durante el embarazo, y por consiguiente no ocurre en mujeres con toxoplasmosis crónica. La transmisión congénita se presenta aproximadamente en el $30 \%$ de las toxoplasmosis maternas recientes, correspondiendo la gran mayoría de los casos a infecciones fetales tardías, que no se manifiestan clínicamente en el día del nacimiento ${ }^{3}$. El riesgo de toxoplasmosis congénita guarda relación con factores epidemiológicos, cuyo estudio permite calcular la incidencia teórica del fenómeno. Las cifras estimativas, elaboradas en diferentes paises, oscilan entre un $0,1 \%$ y un $0,8 \%$; sin embargo, en la actualidad se acepta una frecuencia aproximada de un caso por cada 1.000 nacimientos como valor medio ${ }^{2}$.

El estudio de la madre y el hijo para toxoplasmosis está dirigido, fundamentalmente, a la pesquisa serológica de las embarazadas que se infectan durante el periodo de la gestación, pues el tratamiento inmediato de ellas reduce el riesgo de transmisión transplacentaria en $50 \%{ }^{5}$. Un resulta do serológico sugerente de infección materna recjente, durante el embarazo, sirve además, para seleccionar los casos con riesgo de infección congénita, que requieren estudio inmediato del recién nacido.

El T. cruzi, agente causal de la enfermedad de Chagas, es endémico y se limita al continente americano, donde la parasitosis constituye un importante problema de salud pública en algunos paises6. En Chile el área endémica se extiende desde la I hasta la VI Región. La transmisión congénita de $T$. cruzi puede ocurrir en cualquier periodo de la infección materna y repetirse en embarazos sucesivos?

Se dispone de amplia información sobre la prevalencia de la infección en mujeres que residen en áreas de alta y baja endemia chagásica; sin embargo, aún no se ha podido establecer la incidencia de la transmisión congénita del parásito en relación al total de embarazadas infectadas por $T$. cruzi. Las cifras varian de un lugar a otro, lo que es atribuido fundamentalmente, a factores regionales, cuyo origen constituye un problema de gran actualidad ${ }^{8}$. El estudio sistemático de la tripanosomiasis congénita puede iniciarse en el nacimiento, ya que no tequiere examen serológico de las embarazadas. Sin embargo, la selección previa de embarazadas con evidencia de enfermedad de Chagas puede ser útil, ya que permite limitar el estudio de los recién nacidos a los casos con riesgo de infección congénita.

En Chile se han descrito infecciones congénitas graves, con sintomas "clásicos", producidas por T. gondii ${ }^{9-10}$ y T. cruzi 11- 12 . En la práctica se ha podido apreciar además, la aparición de sintomas tardios y secuelas en el S.N.C. que no corresponden a los tratamientos específicos. Se supone que las infecciones congénitas leves, inaparentes de los recién nacidos, ocurrirían con mayor frecuencia que lo sospechado en el pasado; sin embargo, aún no se dispone de elementos valederos para apoyar djcha hipotesis.

Con los antecedentes expuestos parece importante analizar si la incidencia y el alcance clínico de las infecciones congénitas por $T$. gondii y $T$. cruzi justificarían implantar controles serológicos sistemáticos en embarazadas, recién nacidos 0 ambos en nuestro pais; la factibilidad de realizar dichos estudios en gran escala, con los medios a nuestro alcance y el método más adecuado para realizar dicha tarea.

Para dar respuestas a las incógnitas planteadas se hicieron encuestas sero-epidemiológicas en maternidades de diferentes zonas del país ${ }^{13-14}$. 15. Los resultados y las experiencias obtenidas, constituyen la base del análisis expuesto en este trabajo.

\section{MATERIAL Y METODOS}

El material del análisis proviene de 3 encuestas hechas en las maternidades del Hospital San Juan de Dios de Santiago ${ }^{13}$, del Hospital de Vicuña ${ }^{14}$ y del de Ovalle ${ }^{15}$. En estos trabajos se emplearon diferentes criterios para pesquisar aquellos casos que podían involucrar un riesgo de infección congénita por los parásitos.

En el estudio 1 (Grupo Santiago) primaba el interés por la enfermedad de Chagas, por lo que se inició el estudio en la fecha del nacimiento. El material examinado corresponde a 402 muestras de sangre de cordón, recolectadas al azar, en la Maternidad del Hosp. San Juan de Dios de Santiago.

El estudio 2 (Grupo Vicuña) se planificó siguiendo métodos recomendados para toxoplas. mosis, que incluyen el control previo de las embarazadas. Con estos fines se recolectaron 338 muestras de sangre, correspondientes a $213 \mathrm{em}$ barazadas, no seleccionadas, residentes en el Valle de Elqui, que acudieron al control prenatal en la maternidad del Hospital de Vicuña.

En el estudio 3 (Grupo Ovalle) se ensayó una tercera modalidad en 240 embarazadas tomando 
una sola muestra de sangre obtenida durante el último tercio del período gestacional. Los casos proceden de la maternidad del Hospital de Ovalle (Valle de Limarí).

En los tres estudios se agregaron fichas que incluían datos personales y antecedentes obstétri$\cos$ de las madres, características del parto, examen físico de los recién nacidos e información de interés epidemiológico con respecto a toxoplasmosis y enfermedad de Chagas. Cada estudio comprendió tres etapas con exámenes paralelos para ambas parasitosis.

La primera etapa correspondió a la pesquisa serológica de los casos que podían involucrar riesgo de infección congénita, por $T$. gonđii o $T$. cruzi (Serología indicativa o sospechosa de toxoplasmosis materna reciente o positiva para enfermedad de Chagas). Los exámenes serológicos se realizaron mediante reacción de inmunofluorescencia indirecta (RIFI-IgG), utilizando antigenos de $T$. gondii y $T$. cruzi. En las muestras positivas, para toxoplasmosis con títulos superiores a 1/256 o para enfermedad de Chagas desde la dilución $1 / 10$, se hicieron además RIF[-IgG específicas para el correspondiente parásito.

La segunda etapa está destinada al estudio de los recién nacidos con riesgo de infección congénita, según selección serológica previa. Los exámenes de los niños se iniciaron en lo posible el dia de su nacimiento, con examen físico y RIFI-IgG e IgM, para toxoplasmosis o enfermedad de Chagas, en sangre del cordón.

En los casos con serología positiva para enfer. medad de Chagas, se procedió a buscar el T. cruzi en la sangre de los recjén nacidos, mediante xenodiagnóstico y examen microscópico directo de seis frotis y seis gotas gruesas y xenodiagnósticos.

En los casos sospechosos de toxoplasmosis no se intentó aislar el parásito, por el bajo rendimiento de las técnicas y las dificultades para obtener el material en condicjones adecuadas.

La tercera etapa del estudio comprende los controles progresivos postnatales de los niños con riesgo de infección congénita.

Los recién nacidos con infección parasitológica demostrada en el dia de su nacimiento, fueron sometidos a exámenes clínicos y de laboratorjo, oportunamente tratados y controlados por pediatras familiarizados con el problema.

En los recién nacidos que sólo habian presen. tado serología positiva para toxoplasmosis o enfermedad de Chagas en la sangre del cordón, se practicaron controles serológicos (RIFI-IgG) durante los primeros meses de edad hasta descartar la infección. En algunos casos se hicieron también RIFI-IgM y, se aplicaron nuevamente xenodiagnósticos si procedía.

\section{RESULTADOS}

El estudio serológico selectivo para toxoplasmosis (Tabla 1-A) mostró que la prevalencia de la infección materna alcanzaba a $35,2 \%$ en el grupo estudiado en Santiago y valores levemente inferiores en las mujeres de Vicuña $(29,1 \%)$ y Ovalle $26,3 \%$. La Mayoría de los resultados correspondieron a títulos bajos (1/16 y 1/256); títulos más elevados (1/512 a 1/2048) fueron encontrados en tres casos del grupo de Santiago y en tres de Vicuña. Estos 6 casos fueron considerados con posible riesgo de transmisión congénita y en cinco de ellos pudo practicarse estudios clínico-serológioo, por lo menos parciales (Tabla 1-B).

En el grupo de Santiago, sin control serológico de la embarazada, un nifo se perdió totalmente para el estudio, otro nació sano con IgM negativa y presentó leve descenso de los títulos para la RJFl-IgG en el único control practicado al mes de edad. El tercer niño nació sano; sin embargo, el estudio serológico de la madre, agregado después del parto, presentó resultados indicativos de toxoplasmosis aguda (RIFJ-IgG + 1/64000, RIFl-IgM + 1/64). El njño se desarrolló normal durante los 6 primeros meses de la vida y los anticuerpos desaparecieron paulatinamente durante dicho periodo.

Los tres casos de Vicuña fueron considerados negativos por las siguientes razones: La serologia de las madres, practicada antes y después del parto, indicó persistencia de títujos de 1/1.024 en un caso y descenso de los títulos en los dos restantes. Esto hace poco probable que dichas madres se hubieran infectado durante el embarazo y por consiguiente, no existiría riesgo de transmisión congénita. Los tres niños nacieron sanos, dos fueron controlados hasta los 6 meses, presentando un desarrollo normal y serología negativa en un caso y descenso del título para RIFI-IgG en el otro. El tercero, controlado a los dos meses de edad, mostraba desarrollo normal y marcado descenso de los títulos.

El estudio serológico inicial para enfermedad de Chagas (Tabla $2-A$ ) permitó pesquisar infección por T. cruzi en 11 de 402 casos de Santiago $(2,7 \%), 25$ de 213 de Vicuña $(11,8 \%)$ y en 43 de 240 de Ovalle $(17,9 \%)$.

De los 79 casos con riesgo de infección congé. nita se perdieron del estudio un niño del grupo de Santiago (alta precoz/domicilio falso), dos de Vicuña (traslado a otro lugar) y 5 de Ovalle (por vivir en zonas rurales apartadas de difícil acceso).

La casuística, compuesta de 71 casos, (Tabla 2-B), comprende 37 niños con estudio completo (estudiados al nacer $y$ controlados en perío- 
do post-natal). En 14 R.N. se aplicaron solamente los exámenes fijados para el primer control (al nacer). Seis resultaron serológicamente negativos en sangre del cordón, ellos correspondían a madres con RIFI-IgG positiva al 1/20. En 20 niños, 8 de Vicuna y 12 de 0 valte, que habían nacido en sus domicilios, se aplicaron solamente los controles fijados para el período postnatal.

Se demostró infección por $T$. cruzi, mediante xenodiagnóstico, en 4 casos, en uno de ellos se encontraron, adernás, parásitos en exămenes directos de sangre. Tres de los casos positivos (dos de Santiago y el de Ovalle) correspondieron a hallazgos en fecha del nacimiento, lo que indica. ría su origen congénito. En el caso pesquisado en Vicuña, se trata de un niño que había presentado xenodiagnóstico y RIFI-IgM negativa al nacer, en el cual los xenodiagnósticos aplicados a partir del mes de edad, resultaron positivos. En dicho caso se observó además, persistencia de los títulos positivos para RJFI-IgG, hasta los 8 meses y resultados positivos para RIFI-IgM en las muestras examinadas durante el $7^{\circ}$ y $8^{\circ}$ mes de la vida ${ }^{14}$.

La aplicación seriada de RIFI-IgG, permitió observar la desaparición total de los anticuerpos, durante los primeros meses de la vida, en 58 casos, descartándose el diagnóstico de infección congénita por $T$. cruzi. En los nueve casos restantes, se dispone de estudios parciales cuyos resultados no permiten extraer conclusiones valederas.

Los controles clínicos de los niños positivos correspondientes al estudio de Santiago, revelaron prematurez y alteraciones del líquido céfalo raquídeo en uno y alteraciones electrocardiográficas en el otro. Ambos casos recibieron tratamiento específico, observándose cura parasitolo. gica en uno de ellos ${ }^{13}$.

Los niños positivos pesquisados en Vicuña ${ }^{14}$ $y$ Ovalle ${ }^{15}$ fueron aparentemente sanos y se desarrollaron normalmente durante el primer at̃o de vida.

Tabla 1

Infección por Toxoplasma Gondii en Madres y Recién Nacidos.

\begin{tabular}{|c|c|c|c|c|c|c|c|}
\hline \multirow[b]{2}{*}{ Procedencia } & \multicolumn{4}{|c|}{ A. INFECCION MATERNA } & \multicolumn{3}{|c|}{ B. INFECCION CONGENITA } \\
\hline & Total & $(+)$ & $\begin{array}{l}\mathbf{I g G} \\
>256\end{array}$ & $\begin{array}{c}\text { R.N. } \\
\text { Estudiados }\end{array}$ & $\underset{(+)}{\mathbf{R} . \mathbf{N} .}$ & $(-)$ & E.i. \\
\hline Santiago & 402 & $35,3 \%$ & 3 & 2 & 0 & 1 & 1 \\
\hline Vicuña & 213 & $29 \%$ & 3 & 3 & 0 & 3 & 0 \\
\hline Ovalle & 240 & $26,3 \%$ & 0 & 0 & 0 & 0 & 0 \\
\hline
\end{tabular}

Tabla 2 .

Infección por Tripanosoma Cruzi en Madres y Recién Nacidos.

\begin{tabular}{lcccccc}
\hline & \multicolumn{2}{c}{ A. INFECCION MATERNA } & & & \multicolumn{2}{c}{ B. INFECCION CONGENITA } \\
\hline Procedencia & Total & (+) & $\begin{array}{c}\text { R.N. } \\
\text { Esiudiados }\end{array}$ & $\begin{array}{c}\text { R.N. } \\
(+)\end{array}$ & $(-)$ & E.i. \\
\hline Santiago & 402 & $11-2,7 \%$ & 10 & 2 & 8 & 0 \\
Vicuña & 213 & $25-11,8 \%$ & 23 & $1^{*}$ & 21 & 1 \\
Ovalle & 240 & $43-17,9 \%$ & 38 & 1 & 29 & 8 \\
\hline Total & 855 & 79 & 71 & $3+\% *$ & 58 & 9 \\
\hline
\end{tabular}

E.i. :Estudio incompleto.

* : Infección demostrada al mes de edad. 


\section{DISCUSION}

El estudjo sistemático conjunto de las infecciones congénitas por $T$. gondii y $T$. cruzi, expuesto en el presente trabajo, reveló aspectos diferentes para toxoplasmosis y enfermedad de Chagas, lo que obliga a analizar por separado, si el alcance clínico de dichas parasitosis justificaría la implantación de medidas profilácticas en gran escala, y si sería factible llevarlas a la práctica en nuestro país.

\section{Toxoplasmosis.}

Los resultados obtenidos con respecto a la toxoplasmosis no le dan la apariencia de un problema importante. Sin embargo, la pesquisa de un solo caso de toxoplasmosis materna aguda en un total de 855 pacientes y que en este no se produjera transmisión transplacentaria del parásito, no permite concluir que la toxoplasmosis carece de importancia en nuestro pais: Los resultados serológicos obtenidos en Santiago $(35 \%)$, Vicuña $(29 \%)$ y Ovałle $(26 \%$ ) (Tabla l-A) con. firman la prevalencia observada en dichas regiones ${ }^{16-17}$. Esto significa que un porcentaje relativamente alto de las mujeres en edad fértil, carece de anticuerpos contra $T$. gondii y por consigujente estaría expuesto a primoinfecciones durante el embarazo, que podrían dar origen a infecciones congénitas en $30 \%$ a $40 \%$ de los casos $^{3}$. La importancia clínica de dichas infecciones de los R.N. y los costos que de ello derivan constituye un problema serio, por lo que algunos autores ${ }^{2}$. 3. 4- 18 recomiendan tratar de prevenirlas mediante controles serológicos sistemáticos en las embarazadas. No cabe duda que dicha medida constituye el recurso profiláctico ideal; sin embargo, en la actualidad se cuestiona su utilidad práctica y se recomienda evaluar el beneficio que se pretende obtener en relación con los costos del estudio serológico rutinario de embarazadas, considerando además, los aspectos epidemiológicos propios de cada región y los recursos económicos de la nación ${ }^{19-20-21}$.

El método recomendado para prevenir la toxoplasmosis congénita resulta bueno si se logra aplicar en forma cabal 4 . Esto significa someter a todas las embarazadas a examen serológico al comienzo del embarazo y repetir la prueba ( 1 ó 2 veces) en las que resulten negativas en el primer control. Obviamente, en Chile no se dispone de los medios para aplicar dichas medidas a nivel nacional o regional. Además, existen otros factores que dificultarían la tarea. En primer lugar, en nuestro país, la mayoría de las mujeres no acu. den en forma regular a los controles prenatales. Este hecho, observado en oportunidades anterio- ies y confirmado al aplicar el método en Vicuña, indica que un número importante de casos escaparían a un control completo. El problema se complicaría además, en nuestro medio, por la gran cantidad de mujeres seronegativas que requeririan controles repetidos. Un estudio sistemático de las embarazadas inclujdas en el presente estudio, habría significado practicar controles serológicos adicionales en aproximadamente 606 $70 \%$ de los 855 casos, de los cuales sólo $0.71 \%$ se infectaria durante los 9 meses del embarazo, según estudios epidemiológicos previos ${ }^{17}$. Cabe preguntarse si seria aconsejable practicar miles de reacciones serológicas para pesquisar una infección materna con el fin de aplicar un tratamiento que evitaría la infección congénita en el $50 \%$ de los casos ${ }^{5}$. Además, habria que considerar que un control serológico obligatorio para ka toxoplasmosis podría causar alarma entre las embaraza. $\operatorname{das}^{20}$.

A pesar de haber desestimado la posibilidad de aplicar medidas profilácticas generalizadas para prevenir la toxoplasmosis congénita, aconsejamos recoger la experiencia de los autores extranje$\operatorname{ros}^{2 \cdot 3 \cdot 4 \cdot 18}$ con el fjn de utilizarla en los casos individuales. El estudio serológico de las embarazadas es de indiscutible valor o para detectar las infecciones agudas que constituyen riesgo de infección congénita, y para tranquilizar a las madres cuyos resultados serológicos positivos co. rresponden a infecciones adquiridas antes del período grávido y que no conducen a infección del feto. Además, se detectarian las mujeres seronegativas, a quienes se debería aconsejar que traten de evitar la infección con quistes yo quistes de $T$. gondii mediante medidas profilácticas simples ${ }^{21}$.

Para reducir el problema de la toxoplasmosis congénita en nuestro pais debería intensificarse la búsqueda de infección en los R.N. Esto es válido para los casos poco frecuentes, con síntomas atribuibles a la parasitosis. Además, sería factible incluir el control de la toxoplasmosis en aquellos estudios que se practiquen con respecto a la enfermedad de Chagas y otras infecciones congénitas (Sindrome de TORCH).

E] método recomendado para reconocer precozmente las infecciones congénitas por $T$. gondii consistiría en resumen, en pesquisar los R.N. con anticuerpos contra el T. gondii mediante RIFIIgG u otra técrica equivalente en sangre del cordón o del R.N. (no debe aplicarse reacciones de hemoaglutinación (RHAI), RAD, ni fijaciớn del complemento (RFC) como única reacción); verificar la existencia de anticuerpos de tipo IgM, específicos contra toxoplasma, en las muestras positivas para RIFI-IgG y agregar estudios serológicos de las madres, (RIFI-lgG e lgM). (La 
cuantificación de IgM total tiene escaso valor en estos $\operatorname{casos}^{22}$; practicar exámenes clínicos especializados en los recién nacidos en quienes se sospeche infección $\mathrm{y}$ seguirlos con controles clínicos y serológicos prolongados hasta obtener resultados concluyentes; tratar con medicamentos a todos los R.N. con evidencia de infección, incluso los asintomáticos.

\section{Enfermedad de Chagas}

Las observaciones sobre la transmisión congénita del $T$. cruzi presentan aspectos de interés, que justificarian a nuestro juicio, ampliar este tipo de investigaciones.

El estudio de 855 casos permitió demostrar infecciones por $T$. cruzi en cuatro casos, correspondiendo tres a infecciones congénitas. Esto confirmaría que la enfermedad de Chagas ocurre con mayor frecuencia que la Toxoplasmosis congénita en nuestro pais. De acuerdo con nuestros resultados (Tabla 2), el problema reviste especial importancia en Santiago. En dicho grupo, compuesto de 402 casas con $2,7 \%$ de infecciones maternas, se pesquisaron dos de enfermedad de Chagas en un total de 10 R.N. con riesgo de transmisión congénita. La evolución clínica ob. servada en estos niños y el efecto favorable del tratamiento iniciado oportunamente en uno, de. mostró la utilidad del control sistemático de la enfermedad de Chagas en los R.N. Sería aconsejable repetir este tipo de estudjos en diferentes maternidades de la capital, con el fin de verificar si la incidencia de la transmisión congénita de $T$. cruzi es tan alta como parece desprenderse de este estudio y si existen diferencias entre poblaciones de distintos sectores de la ciudad.

El problema se presentó de manera diferente en las casuísticas de zonas de alta endemia chagásica. Como era de esperar, se demostró una prevalencia alta de infección materna en las 213 embarazadas estudiadas en Vicuña $(11,8 \%$ ) y las 240 de Ovalle $(17,9 \%)$.

Sin embargo, entre los 61 niños estudjados por riesgo de infección congénita, se demostró el parásito en un R.N. el día de su nacimiento (Grupo Ovalle), y en un lactante, al mes de edad (Grupo Vicuña). En ambos casos los niños infectados eran aparentemente sanos y se desarollaron en forma normal durante los primeros meses de la vida. Estos resultados no inducirían a intensificar el estudio rutinario para enfermedad de Chagas en los R.N. de dichas regiones. Sin embargo, las djscrepancias observadas entre los resultados de Santiago (donde la prevalencia de la infección materna es baja y la infección congénita tiene importancia clínica) y lo observado en Vicuña y Ovalie (con un elevado índice de infección chagásica materna, incidencia baja de transmisión congénita y escaso compromiso clínico en los niños infectados), podría tener importancia científica. Estas diferencias regionales confirman lo descrito por autores extranjeros ${ }^{8}, y$ señalar la necesidad de profundizar la experiencia en ambas zonas con el fin de precisar si el problema en discusión se debería a factores relacionados con las cepas de $T$. cruzi ${ }^{8}$ o con factores inmunitarios del huésped.

La pesquisa precóz de las infecciones chagásicas congénitas mediante control serológico rutinario de los R.N., parece factible en nuestro medio. El área para el estudio de la enfermedad de Chagas se limitaria a las zonas endémicas y no abarcaria todo el pais. El total de casos con tiesgo de infección congénita oscilaría entre 2 y $20 \%$ de los casos encuestados, lo que constituiría un material relativamente manejable. La selec. ción de pacientes se haría mediante un examen serológico por caso. De acuerdo con la experiencja recolectada, recomendamos la siguiente metodología para el estudio de la transmisión congérita de $T$. cruzi en embarazadas y recién nacidos: 1a. fase: El estudio serológico selectivo de las embarazadas, mediante RIFI-IgG para enfermedad de Chagas, puede limitarse a una sola muestra, obtenida al comienzo del tercer trimestre del embarazo, tal como se practicó en el estudio de Ovalle.

2a. fase: Para rastrear el $\mathrm{T}$. cruzi en los recién nacidos: el día de su nacimiento, aconsejamos emplear todos los recursos disponibles, recomen. dando especialmente la aplicación de xenodiag. nósticos.

La aplicación de RIFI-IgG en sangre de cordón es útil pues permite confirmar la infección materna y facilita la interpretación de los resultados serológicos que se obtengan en controles posteriores.

La RIFI-IgM constituye un aporte útil al diagnóstico de la infección congénita cuando resulta positiva; sin embargo, una RIFI-IgM negativa no descarta el diagnóstico ${ }^{2} 3$.

3a. fase: Los controles post-natales de los nifros son engorrosos $\mathrm{y}$ el valor de los resultados es discutible.

Obviamente el niño con infección congénita demostrada al nacer, debe ser tratado y controla. do por todo el período que el médico estime necesario.

En los nifios en quienes no se demuestra el $\mathrm{T}$. cruzi en los primeros días de nacidos, los resulta. dos positivos tardíos de RIFl-IgM y xenodiagnósticos, no aseguran la transmisión congénita del parásito.

Las RIFI-IgG en los controles posteriores al nacimiento son útiles para constatar descenso y 
desaparición de los anticuerpos de tipo IgG, transmitidos pasivamente de la madre al hijo. Para interpretar los resultados debe tenerse en cuenta que la persistencia de dichos anticuerpos tiene relación con el nivel de los títulos obtenidos en la sangre del cordón.

Para facilitar la obtención y envío de las muestras de los controles post-natales, recomendamos recoger la sangre de los lactantes con papel filtro ${ }^{24}$.

\section{RESUMEN}

Se discute la utilidad práctica del estudio sistemático para prevenir las infecciones congénitas por Toxoplasma gondii y Trypanosoma cruzi. EJ análisis se basa sobre 855 encuestas seroepidemiológicas, practicadas en las maternidades del Hospital San Juan de Dios de Santiago y de los Hospitales de Vicuña y Ovalle.

El estudio de la toxoplasmosis no permitió demostrar infección congénita. Sin restar valor al problema clínico de la toxoplasmosis congénita, se llega a la conclusión que el estudio sistemático de las embarazadas es demasiado costoso, técnicamente engorroso y de escasa utilidad en nuestro país. Al respecto se recomienda intensificar la pesquisa precoz de las infecciones por $T$. gondii en los RN.

El estudio de la enfermedad de Chagas permitió pesquisar cuatro infecciones por $T$. cruzi, tres de origen congénito. En Santiago, donde la prevaJencia de la infección materna es baja $(2,7 \%)$, se diagnosticó infección congénita en 2 de 10 R.N. correspondientes a madres chagásicas. En Vicuña y Ovalle, donde un elevado porcentaje de las madres se encuentran infectadas $(11,8 \%$ y 17 , $9 \%$ ), se pesquisó una infección congénita entre 61 hijos de madres chagásicas. Las diferencias observadas entre las zonas de alta y baja endemia indican el interés de proseguir el estudio sistemático de la enfermedad de Chagas en embarazadas y R.N. Dicho tipo de investigación es factible en nuestro país. Las encuestas se limitarian a las zonas endémicas. El total de casos con riesgo de infección ( $2 \%$ al $20 \%$ del total estudiado) constituiría una casuística manejable y la pesquisa de los casos de interés se lograría mediante estudio serológico de una muestra por caso.

Se incluye el método recomendado para estudjar ambas infecciones congénitas.

\section{REFERENCIAS}

1. Alford, Ch.: Inmunoglobulin Determinations in the Diagnosis of Fetal Infection. Pediat. Clin. North Am. 1: 99, 1971.
2. Remington, J. and Desmonds, G.: Toxoplasmosis. In Infectious Diseases of Fetus and New Born Infants. USA, Founders Co, 1976.

3. Desmonds, G.: Toxoplasma, Madre e Hijo. Rev. Méd. Chile. 107: 42, 1979.

4. Thalhammer, $0 .:$ Infección Toxoplasmótica Prenatal. Acta Bioquím. Lat. Am. 14: 16I, 1980.

5. Kraeubig, H.: Praeventive Hehandlung der konnatalen Toxoplasmose. Toxoplasmose, praktische Fragen u. Ergehnisse. Ed. G. Thieme, pp. 104-122, 1966.

6. Atios, A.: Enfermedad de Chagas. En "Parasitología Clínica". Atŕas y Neghme, 2a Edit. 1984. Publicaciones Técnicas Mediteriáneo Ltda.

7. Biffencourt, A.L.: Congenital Chagas' disease (Rev.) Am. J. Dis. Child. 130: 97, 1976.

8. Andrade, $S$.: The influence of the Strain of Typanosoma cruzi in placental infections in mice. Transact. Royal Soc, Trop. Med. Hyg. 76, Nr. 1, 1982.

9. Aristia, A.; Martinez, F, Howard, J, y Latorre, M.: Toxoplasmosis connatal activa en un recién nacido Rev. Chil. Pediatr. 25: 501, 1954.

10. Femández, E, R izzardini, $M$; Schuster, A. $y$ Thiermann, E.: Toxoplasmosis congénita: Estudio dínico de 11 casos, incluso 1 gemelo univitelino. Pediatría (Stgo.) 8: 192, 1965 .

11. Howard, J,E.: La enfermedad de Chagas congénita. Monografía. Editorial Universitaria de Chile, 1962.

12. Atias, A,; Rubio, M,; Lolic, M, y Valenzuela, R..- Un nuevo caso de Enfermedad de Chagas congénita. Bol. Chil. Para sitol. 16; 42, 1961.

13. Muñot, P.; Lorca, M.; Thiemmann, E.; Astorga, B.; Atías, A. y Pino $S$.: Transmisión Congénita del T. cruzi Investigación en la maternidad del Hosp. Sn. Juan de Dios de Santiago. Rev. Chil. Pediatr. 53; 22,1982 .

14. Astorga, B.; Thiermann, E.; Lorco, M.; Muñoz, P.: Gamboa, C. y Atías, A.: Estudio sobre Enfermedad de Chagas congénita en zonas endémicas. 1.- Maternidad de Vicusa, Valle del Elqui. Bol. Hosp. San Juan de Dios: 29: 259, 1982.

15. Astorga, B.; Lorca, M.; Atias, A.i Munoz, P. y Thiermionn, E.: Estudios sobre Enfermedad de Chagas congénita en zonas endémicas. 2.- Maternidad de Ovalle, Valle del Limarí. Parasitol. al día. 8: 8,1984 .

16. Niedmann, G.: Thiermenn, E. y Neghme, A.: Toxoplasmosis en Chile. Estado actual de los estudios clínicos y epidemiológicos en Chile. Bol. Chile. Parasitol. 18: 86, 1963.

17. Thiermann, E. y Atias, A.: Infección por Toxoplasma gondii en Chile y estimación del riesgo de infección congénita. Rev. Med. Chile, 106: 677, 1978.

18. Wilson, $C . B, y$ Remington, $J .:$ What can be done to prevent congenital toxoplasmosis? Am. J. Ostet. Gynecol. 138: 357, 1980.

19. Anónimo: Congenital Toxoplasmosis-Lancet, March 15: 578, 1980.

20. Beattie, C.P.: Congenital Toxoplasmosis-Letters to the Editor, Lancet April 19: 873, 1980 .

21. Frenkel, J, $\boldsymbol{K}$.: Congenital toxoplasmosis: Prevention or palliation? Am. J. Obstet. Gynecol. 14: 359, 1981.

22. Lorca, J.; Lema, G.; Retamal, C.; Lorca, M. y Thiermann, E.: Determinación de inmunoglobulinas - M en 129 recién nacidos normales de Santiago. Rev. Chil. Pediatr. 52: 224, 1981.

23. Lorca, M. y Thiermann, E.: Valor diagnóstico de la Inmunofluorescencia Indirecta con anti-IgM para enfermedad de Chagas en adultos y recién nacidos. 
Rev. Chil. Pediatr. 53: 199, 1982.

24. Lorca, $M .:$ E1 empleo de la muestra de sangre en papel filtro para el estudio seroepidemiológico de la parasitosis. Parasitologia al Día (por publicar). 